\title{
Instantons in Two-Dimensional Noncommutative U(1) Gauge Theory
}

\section{Wolfgang Frisch}

Atominstitut, Vienna University of Technology, Austria

E-mail: w.frischegmx.at

\section{Harald Grosse}

Department for Theoretical Physics, University of Vienna, Austria

E-mail: harald.grossedunivie.ac.at

\section{Harald Markum *广}

Atominstitut, Vienna University of Technology, Austria

E-mail: markumetuwien.ac.at

Theories with noncommutative space-time coordinates represent alternative candidates of grand unified theories. We discuss $\mathrm{U}(1)$ gauge theory in 2 dimensions on a lattice with $\mathrm{N}$ sites. The mapping to a $\mathrm{U}(\mathrm{N})$ one-plaquette model in the sense of Eguchi and Kawai can be used for computer simulations. We are discussing the formulation and evaluation of topological objects. We performed quantum Monte Carlo simulations and calculated the topological charge for different matrix sizes and several values of the coupling constant. We constructed classical gauge field configurations with large topological charge and used them to initialize quantum simulations. It turned out that the value of the topological charge is decreasing during a Monte Carlo history. Our results show that the topological charge is in general supressed. The situation is similar to lattice QCD where quantum gauge field configurations are topologically trivial and one needs to apply some cooling procedure on the gauge fields to unhide the integer number of the instantons.

The XXV International Symposium on Lattice Field Theory

July 30 - August 42007

Regensburg, Germany

\footnotetext{
* Speaker.

${ }^{\dagger}$ To my father
} 


\section{Motivation}

In noncommutative geometry, where the coordinate operators $\hat{x}_{\mu}$ satisfy the commutation relation $\left[\hat{x}_{\mu}, \hat{x}_{v}\right]=i \theta_{\mu \nu}$, a mixing between ultraviolet and infrared degrees of freedom takes place [1]]. So lattice simulations are a promising tool to get deeper insight into noncommutative quantum fi eld theories. In this work we have studied noncommutative $\mathrm{U}(1)$ gauge theory on a two-dimensional torus. The advantage of this theory is that there exists an equivalent matrix model which makes numerical calculations feasible [2].

The main topic of the underlying contribution is to study the topological charge in twodimensional noncommutative U(1) gauge theory. The instanton confi gurations carry a topological charge $q$ which is non-integer in this case [3]. We performed Monte Carlo simulations with different values of the coupling constant $\beta$ and looked at the topological charge $q$ in the equilibrium 㕧.

\section{Topology and Instantons in QCD}

The Lagragian of pure gluodynamics (the Yang-Mills theory with no matter fi elds) in Euclidean spacetime can be written as

$$
\mathscr{L}=\frac{1}{4 g^{2}} G_{\mu \nu}^{a} G_{\mu \nu}^{a}
$$

where $G_{\mu \nu}^{a}$ is the gluon fi eld strength tensor

$$
G_{\mu \nu}^{a}=\partial_{\mu} A_{v}^{a}-\partial_{v} A_{\mu}^{a}+f^{a b s} A_{\mu}^{b} A_{v}^{c}
$$

and $f^{a b s}$ are structure constants of the gauge group considered. The classical action of the YangMills fi elds can be identically rewritten as

$$
S=\frac{1}{8 g^{2}} \int d x^{4}\left(G_{\mu \nu}^{a} \pm \tilde{G}_{\mu \nu}^{a}\right)^{2} \mp \frac{8 \pi^{2}}{g^{2}} Q
$$

where Q denotes the topological charge

$$
Q=\frac{1}{32 \pi^{2}} \int d x^{4} G_{\mu \nu}^{a} \tilde{G}_{\mu \nu}^{a}
$$

with

$$
\tilde{G}_{\mu \nu}^{a}=\frac{1}{2} \varepsilon_{\mu v \alpha \beta} G_{\alpha \beta}^{a}
$$

\section{Definition of the Topological Charge in Two Dimensions}

\subsection{Lattice Regularization of Noncommutative Two-Dimensional U(1) Gauge Theory}

The lattice regularized version of the theory can be defi ned by an analog of Wilson's plaquette action

$$
S=-\beta \sum_{x} \sum_{\mu<v} U_{\mu}(x) \star U_{v}(x+a \hat{\mu}) \star U_{\mu}(x+a \hat{v})^{\dagger} \star U_{v}(x)^{\dagger}+\text { c.c. }
$$


where the symbol $\hat{\mu}$ represents a unit vector in the $\mu$-direction and we have introduced the lattice spacing $a$. The link variables $U_{\mu}(x)(\mu=1,2)$ are complex fi elds on the lattice satisfying the starunitarity condition. The star-product [1] on the lattice can be obtained by rewriting its defi nition within noncommutatiuve derivatives in terms of Fourier modes and restricting the momenta to the Brillouin zone.

Let us defi ne the topological charge for a gauge fi eld confi guration on the discretized twodimensional torus. In the language of fi elds, we defi ne the topological charge as

$$
q=\frac{1}{4 \pi i} \sum_{x} \sum_{\mu v} \varepsilon_{\mu v} U_{\mu}(x) \star U_{v}(x+a \hat{\mu}) \star U_{\mu}(x+a \hat{v})^{\dagger} \star U_{v}(x)^{\dagger}
$$

which reduces to the usual defi nition of the topological charge in $2 \mathrm{~d}$ gauge theory

$$
q=\frac{1}{4 \pi} \int d^{2} x \varepsilon_{\mu v} G_{\mu v}
$$

in the continuum limit.

\subsection{Matrix-Model Formulation}

It is much more convenient for computer simulations to use an equivalent formulation, in which one maps functions on a noncommutative space to operators so that the star-product becomes nothing but the usual operator product, which is noncommutative. The action (3.1) can then be written as

$$
\begin{aligned}
S & =-N \beta \sum_{\mu \neq v} \operatorname{tr}\left\{\hat{U}_{\mu}\left(\Gamma_{\mu} \hat{U}_{v} \Gamma_{\mu}^{\dagger}\right)\left(\Gamma_{v} \hat{U}_{\mu}^{\dagger} \Gamma_{v}^{\dagger}\right) \hat{U}_{v}^{\dagger}\right\}+2 \beta N^{2} \\
& =-N \beta \sum_{\mu \neq v} \mathscr{Z}_{v \mu} \operatorname{tr}\left(V_{\mu} V_{v} V_{\mu}^{\dagger} V_{v}^{\dagger}\right)+2 \beta N^{2}
\end{aligned}
$$

where $V_{\mu} \equiv \hat{U}_{\mu} \Gamma_{\mu}$ is a $\mathrm{U}(N)$ matrix with $N$ the linear extent of the original lattice. An explicit representation of $\Gamma_{\mu}$ in the $d=2$ case shall be given in Sec. 5. This is the twisted Eguchi-Kawai (TEK) model [5], which appeared in history as a matrix model equivalent to the large $N$ gauge theory [6]. We have added the constant term $2 \beta N^{2}$ to what we would obtain from (3.1) in order to make the absolute minimum of the action zero.

By using the map between fi elds and matrices, the topological charge 3.2) can be represented in terms of matrices as

$$
\begin{aligned}
q & =\frac{1}{4 \pi i} N \sum_{\mu v} \varepsilon_{\mu v} \operatorname{tr}\left\{\hat{U}_{\mu}\left(\Gamma_{\mu} \hat{U}_{v} \Gamma_{\mu}^{\dagger}\right)\left(\Gamma_{v} \hat{U}_{\mu}^{\dagger} \Gamma_{v}^{\dagger}\right) \hat{U}_{v}^{\dagger}\right\} \\
& =\frac{1}{4 \pi i} N \sum_{\mu v} \varepsilon_{\mu v} \mathscr{Z}_{v \mu} \operatorname{tr}\left(V_{\mu} V_{v} V_{\mu}^{\dagger} V_{v}^{\dagger}\right)
\end{aligned}
$$

\section{Numerical Results for the Topological Charge in the TEK Model}

We have computed the topological content of gauge fi eld confi gurations produced by quantum Monte Carlo simulations. In Fig. 1 we display scatter plots of the action $S$ without a factor of 

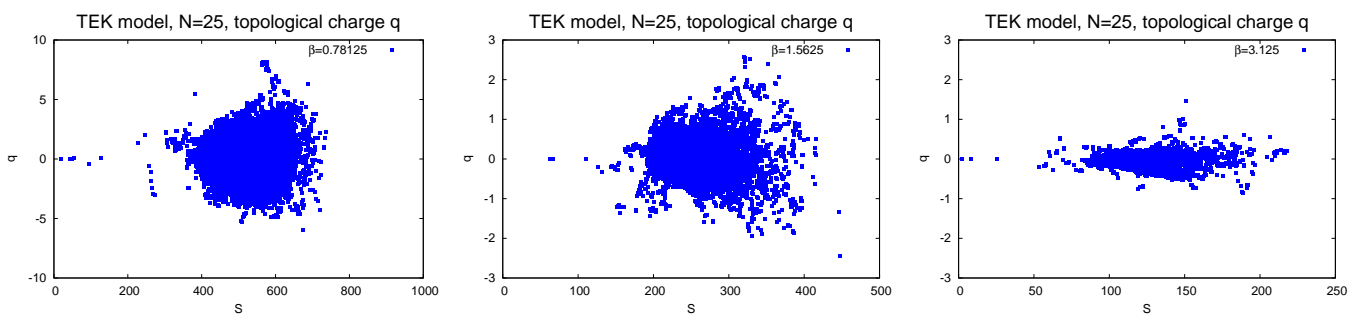

Figure 1: Scatter plots of the action $S$ divided by $\beta$ (x-axis) and the topological charge $q$ (y-axis) for a Monte Carlo simulation (cold start) at $N=25$ and $\beta=0.78125,1.5625$ and 3.125.
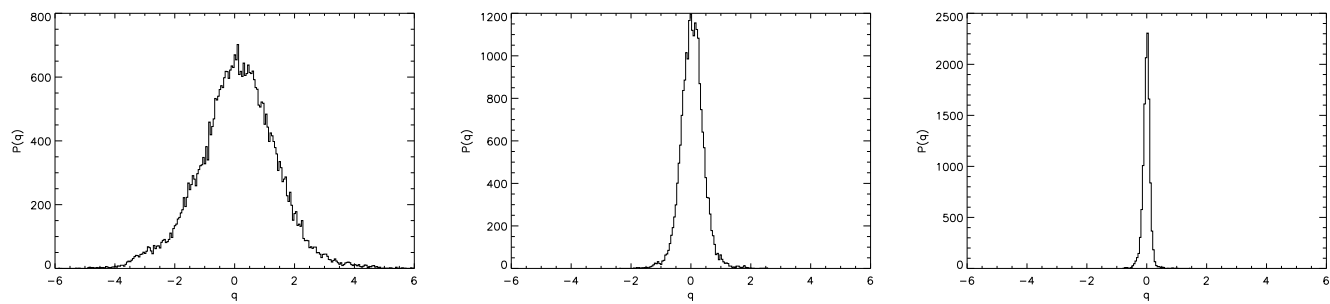

Figure 2: Distribution $P$ of the topological charge $q$ in the Twisted Eguchi-Kawai model for $N=25$ and $\beta=0.78125,1.5625$ and 3.125 .

$\beta$ in its defi nition Eq. 3.5) and the topological charge $q$ performing a cold start. The size of the matrix is $N=25$ and the values of the coupling $\beta$ are chosen to yield a non-commutativity parameter $\theta=2.55,1.27,0.63$, respectively. One observes a decrease of the action with increasing $\beta$ due to stronger coupling of the matrices in analogy to lower temperature in an Ising model. The importance sampling of the system with smaller action generates smaller values of its topological content. This can also be seen from the distributions of the topological charge in Fig. 2 where the peaks become narrower with increasing $\beta$. Similar plots have been obtained for a larger matrix size $N=35$, for more results see Ref. [Њ1. To compare the topology-action diagrams on the same scale, we display in Fig. 3 our simulation for $N=25$ with all $\beta$-values considered in a single plot.

\section{Classical Solutions}

The classical equation of motion can be obtained from the action (3.5) as [7]

$$
V_{\mu}^{\dagger}\left(W-W^{\dagger}\right) V_{\mu}=W-W^{\dagger}
$$

where the unitary matrix $W$ is defi ned by

$$
W=\mathscr{Z}_{v \mu} V_{\mu} V_{v} V_{\mu}^{\dagger} V_{v}^{\dagger}
$$




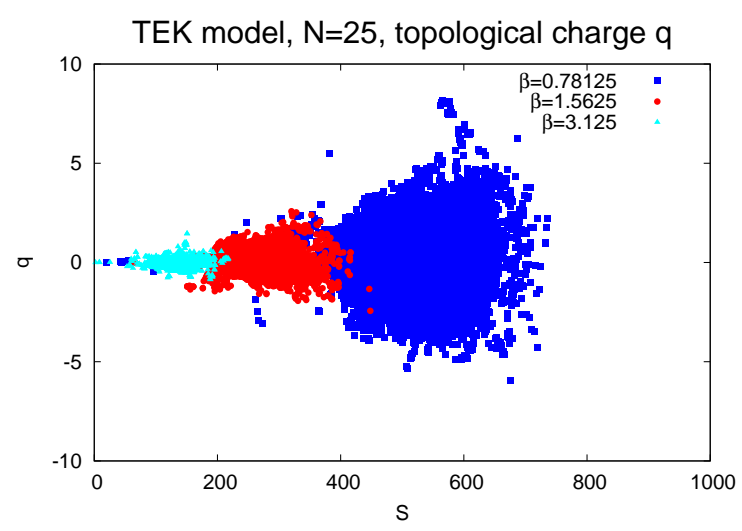

Figure 3: Scatter plots of the action $S$ divided by $\beta$ (x-axis) and the topological charge $q$ (y-axis) for the Monte Carlo simulations at $N=25$ and $\beta=0.78125,1.5625$ and 3.125, combining different couplings.

The general solutions to this equation can be brought into a block-diagonal form [7]

$$
V_{\mu}=\left(\begin{array}{cccc}
\Gamma_{\mu}^{(1)} & & & \\
& \Gamma_{\mu}^{(2)} & & \\
& & \ddots & \\
& & & \Gamma_{\mu}^{(k)}
\end{array}\right)
$$

by an appropriate $\mathrm{SU}(N)$ transformation, where $\Gamma_{\mu}^{(k)}$ are $n_{k} \times n_{k}$ unitary matrices satisfying the 't Hooft-Weyl algebra

$$
\begin{aligned}
\Gamma_{\mu}^{(j)} \Gamma_{\nu}^{(j)} & =Z_{\mu \nu}^{(j)} \Gamma_{\nu}^{(j)} \Gamma_{\mu}^{(j)} \\
Z_{12}^{(j)} & =Z_{21}^{(j) *}=\exp \left(2 \pi i \frac{m_{j}}{n_{j}}\right) \\
m_{j} & =\frac{n_{j}+1}{2}
\end{aligned}
$$

An explicit representation is given, for instance, by the clock and shift operators, $Q$ and $P$

$$
\Gamma_{1}^{(j)}=P_{n_{j}}, \quad \Gamma_{2}^{(j)}=\left(Q_{n_{j}}\right)^{m_{j}}
$$

For each solution, the action and the topological charge can be evaluated as

$$
\begin{aligned}
& S=4 N \beta \sum_{j} n_{j} \sin ^{2}\left\{\pi\left(\frac{m_{j}}{n_{j}}-\frac{M}{N}\right)\right\} \\
& q=\frac{N}{2 \pi} \sum_{j} n_{j} \sin \left\{2 \pi\left(\frac{m_{j}}{n_{j}}-\frac{M}{N}\right)\right\}
\end{aligned}
$$

Note that the topological charge $q$ is not an integer in general. If we require the action to be less than of order $N$, however, the argument of the sine has to vanish for all $j$. In that case the topological charge approaches an integer

$$
q \simeq N\left(\sum_{j} m_{j}-M\right)
$$

which is actually a multiple of $N$. 


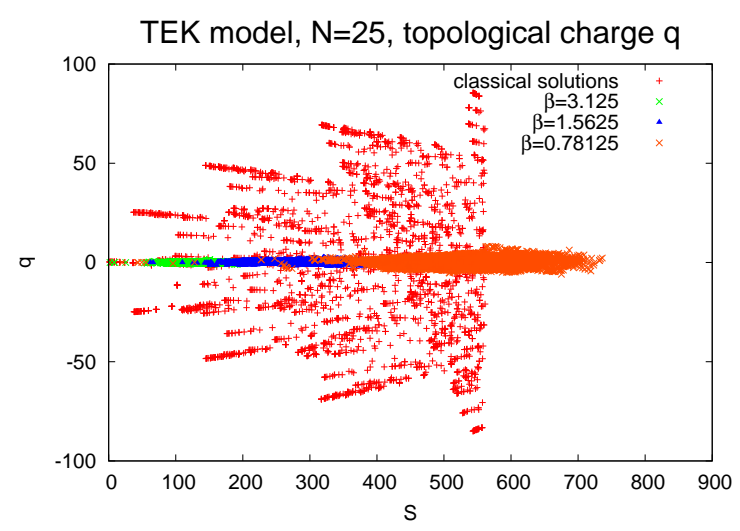

Figure 4: Scatter plots of the action $S$ divided by $\beta$ (x-axis) and the topological charge $q$ (y-axis) for Monte Carlo histories at $N=25$ with cold starts being topologically trivial, $q=0$. The numbers of the classical topological charges are superimposed.
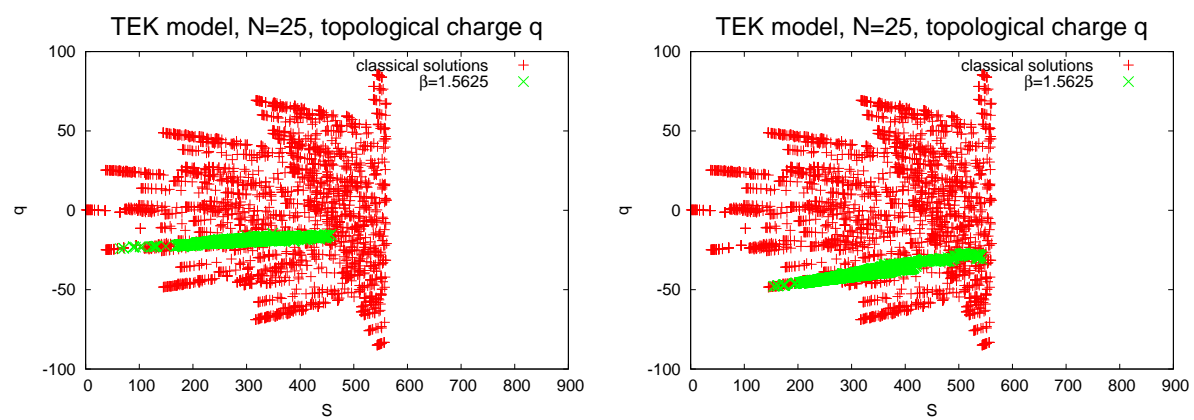

Figure 5: Scatter plots of the action $S$ divided by $\beta$ (x-axis) and the topological charge $q$ (y-axis) for Monte Carlo histories at $N=25$ and $\beta=1.5625$ with starts at $q=-25$ and -50 . The numbers of the classical topological charges are superimposed.

\section{Comparison of Quantum Monte Carlo and Classical Solutions}

In the following analysis we compare the classical topological charges taken from Ref. [B] with our quantum Monte Carlo simulation at $N=25$. In Fig. 7 we plot our data for a cold start from Fig. 3 together with the classical solutions. One sees from the scatter plots that the quantum simulation reaches only small topological numbers. This brings the situation of QCD into mind where one has to apply some cooling or smoothing procedure to damp the quantum fluctuations and get in touch with the integer-valued topological charges. Since a confi guration from a cold start is topologically trivial, we constructed classical solutions and started with them. In Fig. D we overlay the Monte Carlo histories at $\beta=1.5625$ starting with $q=-25$ and $q=-50$, respectively, to the scatter plots of the classical topological charges from Ref. [3]. One observes that the equilibrium confi gurations tend to smaller values of $q$. Remarkably, the equilibration seems to proceed along a "classical branch". 


\section{Conclusion and Outlook}

The diagram of the classical topological charges and the corresponding action from Ref. [3] allows for much larger values of $q$. The equilibrium confi gurations visit only a small part of this charge-action diagram. Thus we constructed classical gauge fi eld confi gurations with large topological charge and used them as start confi guration for quantum simulations. It turned out that the value of the topological charge is decreasing during a Monte Carlo history, preferably along the classical minima. To summarize, our results show that the topological charge is in general supressed.

The situation is reminiscent of lattice QCD where quantum gauge fi eld confi gurations are topologically trivial and one needs to apply some smoothing procedure on the gauge fi elds to unhide instantons. It would be interesting to adapt cooling techniques from QCD to the two-dimensional noncommutative $\mathrm{U}(1)$ theory. At present we are working on this. Even more desirable would be to tackle the four-dimensional noncommutative gauge theory in order to obtain a realistic comparison of its topological content with the well-studied topological objects like instantons and monopoles in QCD.

\section{References}

[1] R.J. Szabo, Quantum Field Theory on Noncommutative Spaces, Phys. Rept. 378 (2003) 207 [hep-th/0109162];

R.J. Szabo, Discrete Noncommutative Gauge Theory, Mod. Phys. Lett. A16 (2001) 367 [hep-th/0101216].

[2] W. Bietenholz, F. Hofheinz, J. Nishimura, Non-Commutative Field Theories beyond Perturbation Theory, Fortsch. Phys. 51 (2003) 745 [hep-th / 0212258];

W. Bietenholz, F. Hofheinz, J. Nishimura, Y. Susaki, J. Volkholz, First Simulation Results for the Photon in a Non-Commutative Space, Nucl. Phys. Proc. Suppl. 140 (2005) 772

[hep-lat/0409059];

W. Bietenholz, A. Bigarini, F. Hofheinz, J. Nishimura, Y. Susaki, J. Volkholz, Numerical Results for U(1) Gauge Theory on $2 d$ and $4 d$ Non-Commutative Spaces, Fortsch. Phys. 53 (2005) 418 [hep-th/0501147].

[3] H. Aoki, J. Nishimura, Y. Susaki, The Index of the Overlap Dirac Operator on a Discretized $2 d$ Non-Commutative Torus, J. High Energy Phys. 02 (2007) 033 [hep-th / 0602078 ];

H. Aoki, J. Nishimura, Y. Susaki, Suppression of Topologically Nontrivial Sectors in Gauge Theory on $2 d$ Non-Commutative Geometry [hep-th/ 0604093 ].

[4] W. Frisch, Simulation and Topology of Two-Dimensional Noncommutative U(1) Gauge Theory, Diploma Thesis, Vienna University of Technology (Vienna 2007).

[5] A. González-Arroyo, M. Okawa, A Twisted Model for Large-N Lattice Gauge Theory, Phys. Rev. D27 (1983) 2397.

[6] T. Eguchi, H. Kawai, Reduction of Dynamical Degrees of Freedom in the Large-N Gauge Theory, Phys. Rev. Lett. 48 (1982) 1063.

[7] L. Griguolo, D. Seminara, Classical Solutions of the TEK Model and Noncommutative Instantons in Two Dimensions, J. High Energy Phys. 03 (2004) 068 [hep-th/ 0311041$].$ 